\title{
Active Mobile Phone Detector using Radio Frequency Signal
}

\section{T.Agalya ${ }^{1} \mid$ R.Mathumitha ${ }^{1} \mid$ P.Poongodi ${ }^{1} \mid$ D.Sangeetha ${ }^{1} \mid$ K.S.Gowthaman ${ }^{2}$}

${ }^{1}$ UG Scholar, Department of EEE, Government College of Engineering, Sengipatti, Thanjavur-613402, Tamilnadu, India. ${ }^{2}$ Assistant Professor, Department of EEE, Government College of Engineering, Sengipatti, Thanjavur-613402, Tamilnadu, India.

To Cite this Article

T.Agalya, R.Mathumitha, P.Poongodi, D.Sangeetha and K.S.Gowthaman, “Active Mobile Phone Detector using Radio Frequency Signal", International Journal for Modern Trends in Science and Technology, Vol. 06, Issue 07, July 2020, pp.:96-99; https://doi.org/10.46501/IJMTST060715

\section{Article Info}

Received on 14-June-2020, Revised on 22-June-2020, Accepted on 29-June-2020, Published on 16-July-2020.

\section{ABSTRACT}

Mobile phone has made our communication easier. It is used for sending and receiving text messages, calls, E-mails, games, camera. Mobile phones are said to be in active mode, when they receive calls or make calls, sending and receiving text messages and when it is connected to internet. Some people uses mobile phone in unnecessary places where mobile phones are prohibited. Mobile phone detector is a device used to detect the active mobile phone in unauthorized places. It receives the radio frequency signals from mobile phones through antenna and indicates the use of cell phones by LED and buzzer. This device is usefulfor detecting the use of mobile phone in examination hall, during meeting and plane stations. It can also be used in military for security purpose and also in places where use of mobile phones are prohibited.

KEYWORDS: op-amp[ICLM358], piezo Buzzer, RF signal, Antenna

Copyright (C) 2020 International Journal for Modern Trends in Science and Technology

DOI: https://doi.org/10.46501/IJMTST060715

\section{INTRODUCTION}

In recent years our communication style was more advanced and people use mobile phone in unauthorized places where use of mobile phones are prohibited. It is very important to detect the use of mobile phones in those places.

There are many ways to avoid the use of mobile phones in unnecessary places. One of these is mobile phone jammer. It ceases the signal reaching the cell phone from base station. It is the good way to avoid the use of mobile phones while meeting and some other places where use of mobiles are restricted. But it also has the disadvantage in the use of jammer. We can't make calls in case of emergency issues.
Thus, mobile phone detector is introduced it has the ability to make and receive calls and can send and receive messages. But during the transmission of signals the LED gets blinking, buzzer keeps beeping.

Mobile phone signal has the transmission frequency of 0.9 to $3 \mathrm{GHz}$ with wavelength of 3.3 to $10 \mathrm{~cm}$. The device mobile phone detector detects the radio frequency signal through antenna when the mobile phone is active. The signals transmit in the form of sine wave and it is received by the antenna.

\section{LITRATURE SURVEY}

The existing detector device mostly discrete components which are more expensive and its 
design is based on down convertor in conjunction with band pass filter. It is not useful because it is inaccurate and expensive [1].

The radio frequency detector using $\mathrm{LC}$ circuit is hard to construct. It is affordable but requires precision tuning. It is used to detect low frequency radiation in AM and FM bands [2].

The another technique is affordable but it is also has the disadvantage of detecting the mobile signal randomly and did not detect the phone accurately [3].

The use of intelligent mobile phone detector is to detect the use of cell phone in restricted area. It detects the GSM signal from the mobile and it will turn on another device which will jam the user's GSM signal [4].

\section{ISSUES IN THE EXISTING}

There are two popular technologies in mobile phone detection which are produced by Berkeley variation system and mobile security products.Both has its own short comings that Berkeley has the disadvantage of detecting the mobile phone only after twenty minutes of its usage. It is the major drawback.

Mobile security product has the disadvantage of detecting mobile phones randomly but not accurately.Most of the detector circuit detects the signal only within the short radius. It will be the major disadvantage of those circuits.

\section{PROPOSED METHODOLOGY}

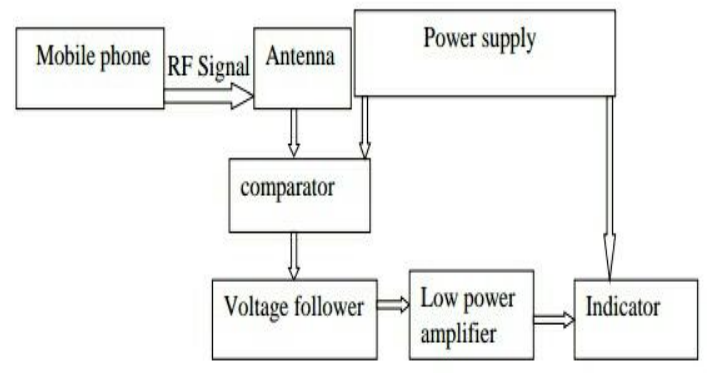

Fig 4.1 Block diagram

In the proposed methodology of our project the circuit is powered by $9 \mathrm{v}$ battery. Then the antenna detects the RF signal when the user send are receive messages and make are receive call. Then the op-amp LM358 amplifies the received RF signal. The op-amp act as the comparator and voltage follower. The amplified signal triggers the buzzer along and make the LED blinks until the signal ceases through the transistor. The transistor act as a low power amplifier.

\section{CIRCUIT DIAGRAM}

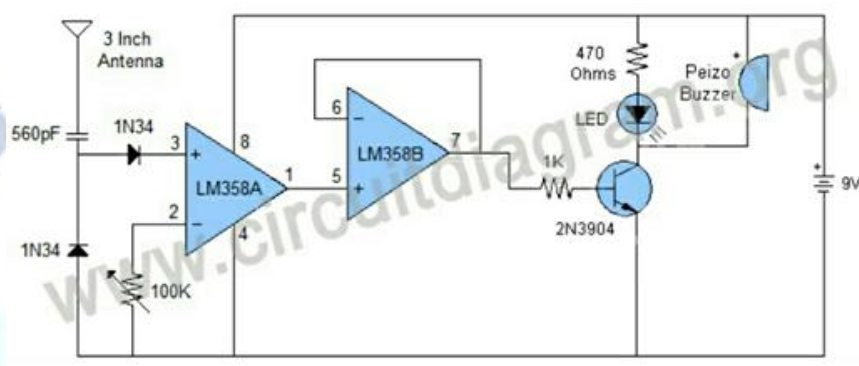

Fig 5.1 circuit diagram

The antenna is used to detect the RF signal from the mobile phone if any signal is transmitted or received. The capacitor carries energy. The RF signal from the phone will disturb the field and forces the capacitor to release energy this is applied to the op-amp through forward biased diode. The output of the LM358 is applied to transistor through high value of resistor. The LED and buzzer drived by the transistor. The battery supply is applied to the supply of the op-amp. The transmission and receiving signals is captured by the antenna the LED will glow and Buzzer make a sound.

\section{COMPONENTS DESCRIPTION}

\subsection{ICLM358}

LM358 is a dual op-amp IC integrated with two op-amps powered by a common power supply. It can be considered as one half of LM324 op-amp which contains four op-amps with common power supply. The differential input voltage range can be equal to that of power supply voltage.

\subsection{TRANSISTOR}

The 2N3904 is a common NPN bipolar junction transistor used for general purpose low-power amplifying or switching applications. It is designed for low current and power, medium voltage, and can operate at moderately high speeds.

\subsection{DIODE}

$1 \mathrm{~N} 34 \mathrm{~A}$ is a Germanium diode. It is appoint contact diode in a package employing $\mathrm{N}$-form Germanium and gives an efficient and excellent linearity when used in image detection, FM detection, radio AM detection.

\subsection{CAPACITOR}


Here we use 560pf capacitor. It is the ceramic capacitor, used to filter out AC ripples. It provide a high level capacity coupled with a smaller size at low price as compared with other lower value capacitor.

\subsection{LED}

A light emitting diode is a semiconductor device that emits light when an electric current is passed through it. Light is produced when the particles that carry the current is combine together in the semiconductor material.

\subsection{BUZZER}

The buzzer consists of an outside case with pins attached to power and ground. Inside a piezo element, which consists of a central ceramic disc surrounded by a metal vibration disc. When current is applied to the buzzer it causes the ceramic disk to contact or expand.

\subsection{POWER SUPPLY}

$9 \mathrm{v}$ battery is used as a power supply in the circuit. In the two electrode one acts as cathode which is the positive end of battery where the current leaves during discharge and another end acts as anode which is the negative side where the current enters during discharge.

\section{EXPERIMENTAL PROTOTYPE}

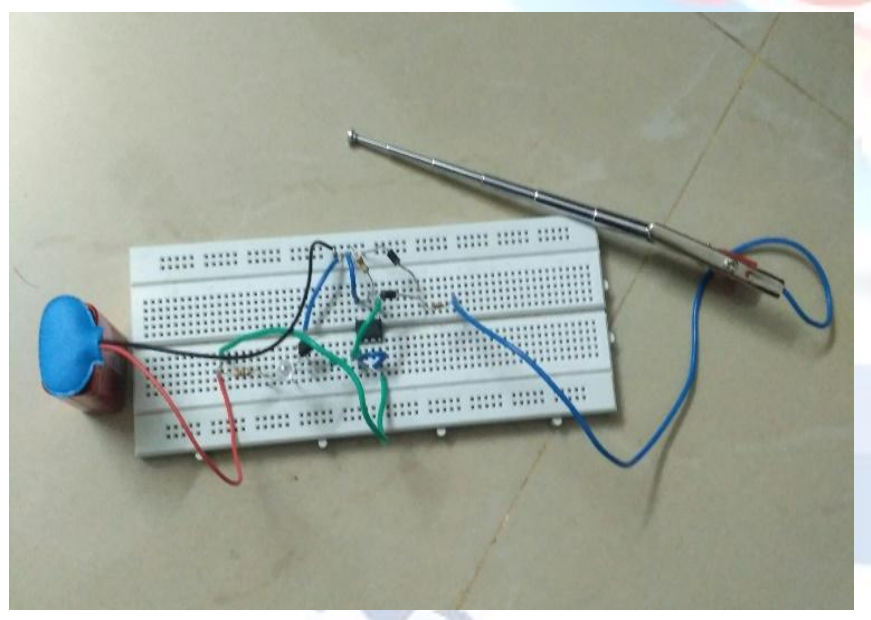

Fig 7.1 Experimental prototype

\section{OUTPUT}

The output of the circuit is discussed here. When the mobile phone is in active mode that is send or receive messages and calls the radio frequency signal is detected by the detector and the detection is indicated by glowing LED and beeping of buzzer.

\subsection{CIRCUIT WHEN THE MOBILE PHONE SIGNAL IS DETECTED}

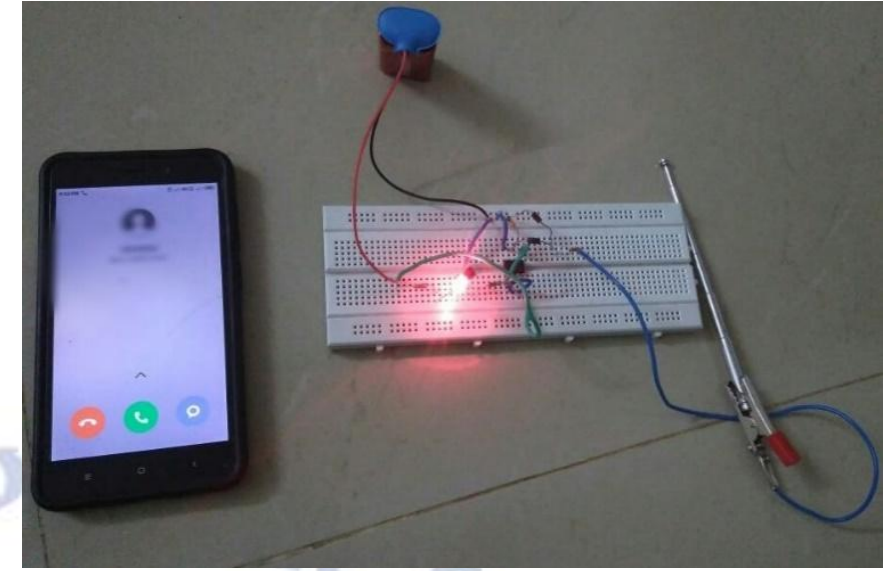

Fig 8.1 circuit when the signal is detected

\subsection{CIRCUIT WHEN THE MOBILE PHONE SIGNAL IS NOT DETECTED}

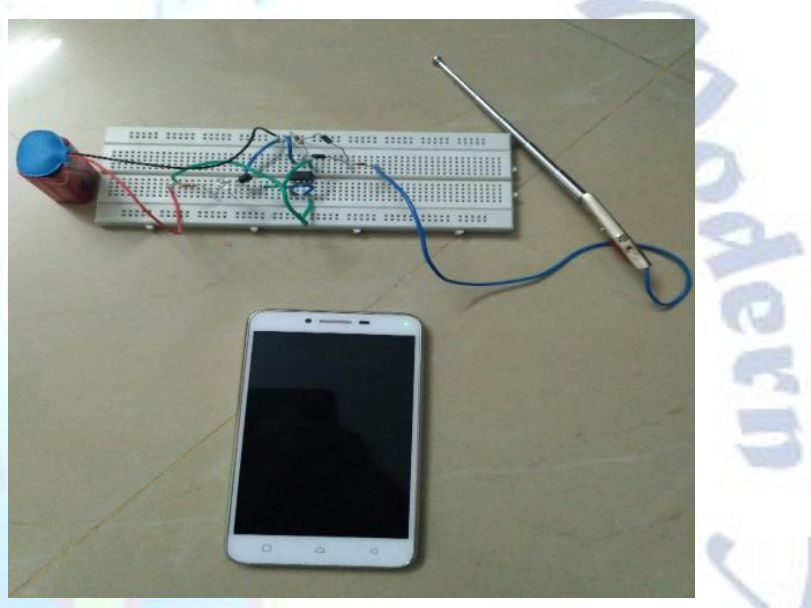

Fig 8.2 circuit when the signal is not detected

\section{Conclusion}

In this project we made an attempt to design a handy, pocket size mobile phone detector, when the mobile phone is in active mode that is when it send or receive calls and messages even the mobile is in silent mode. We designed a circuit which detects the mobile frequency at the distance of 10 to 12 meters. The detection is indicated by glowing of LED and beeping of buzzer. This project is useful for the places where use of mobile phones are prohibited such as private meetings, examination halls, defense establishments, plane stations and military purpose for the sake of security

\section{REFERENCES}

[1] Design and development of mobile phone signal detector(2014) by A.A. Ajasa, O. Shoewu, P. O. Nwamina

[2] Hidden Active cellphone detector(2013) by Anurag Saxena, Dr. Deepak Sharma 
[3] Wholfhound Cell phone Detector, Berkeley Varitronicssystems.2010.Accesed March,2009 Website:http;//www.Bysystems.com

[4] Intelligent cell phone detector system at $4 \mathrm{G}$ bands of frequencies (2017) by Himanshu Verma, Rudra DEV Tiwari, Sarvesh Mishra, Amit Singh, Shubham Srivastava.

[5] College Attendance Management System with Mobile Phone Detector (2018) by MussaveerTungal.

[6] Mobile Phone Detector using OP- AMP(2018) by Manivannan. P, Soniya Jenifer. A, Reeva Princy. S

"Cell phone Detector", Circuit-Project.com

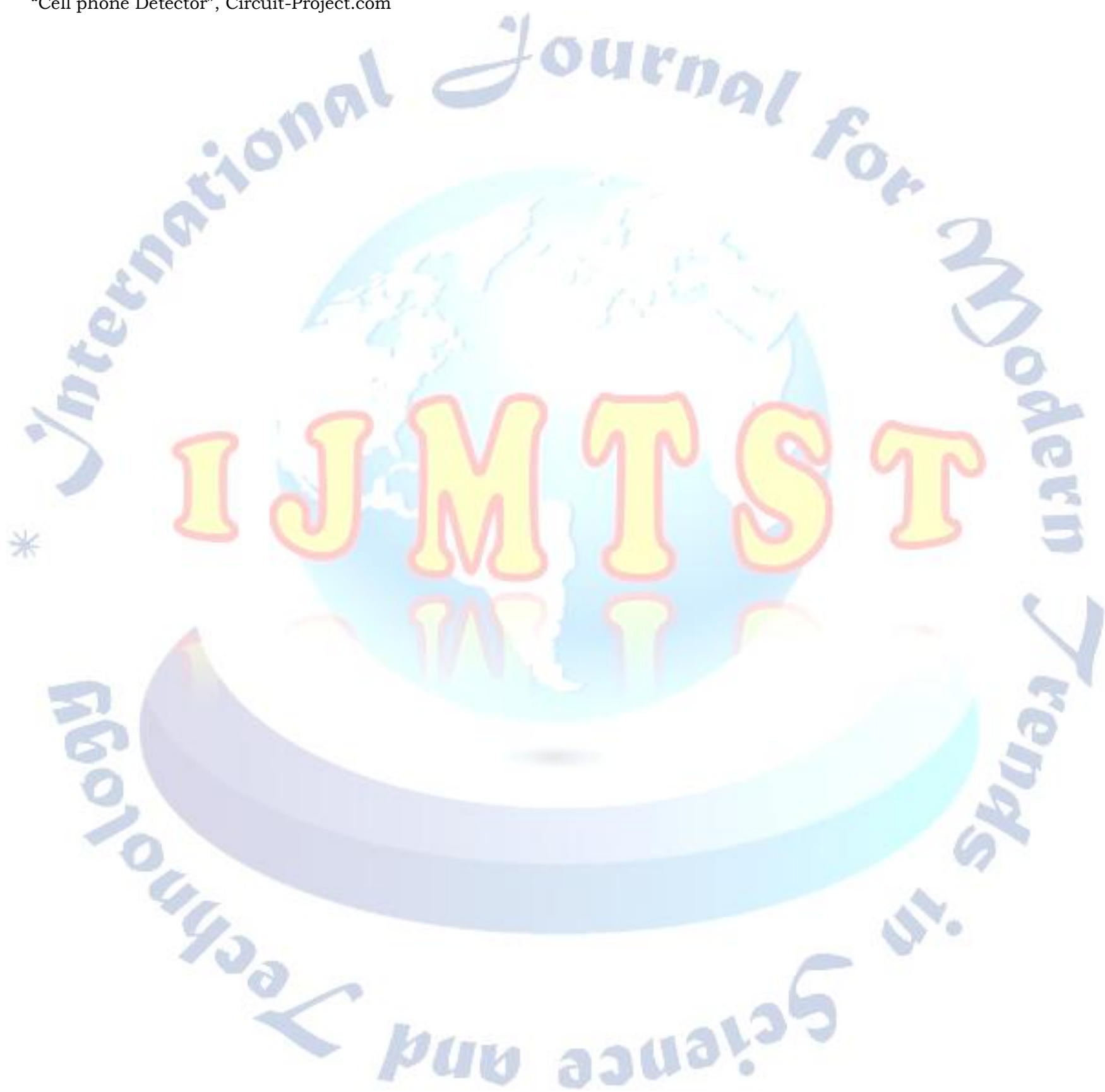

